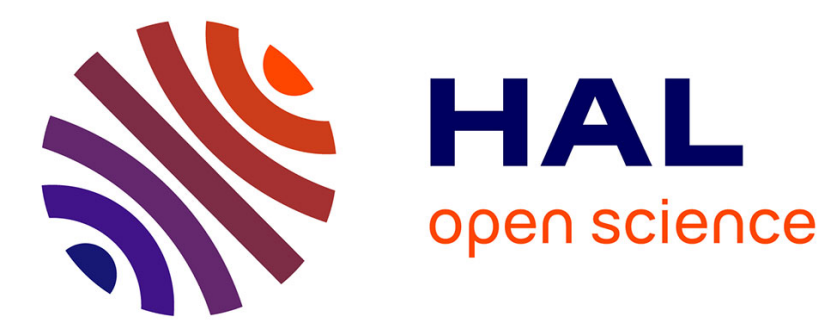

\title{
A new revised desired compensation adaptive control for enhanced tracking: application to RA-PKMs
}

\author{
Moussab Bennehar, Ahmed Chemori, François Pierrot
}

\section{To cite this version:}

Moussab Bennehar, Ahmed Chemori, François Pierrot. A new revised desired compensation adaptive control for enhanced tracking: application to RA-PKMs. Advanced Robotics, 2016, 30 (17-18), pp.1199-1214. 10.1080/01691864.2016.1204248 . hal-01346433

\section{HAL Id: hal-01346433 \\ https://hal.science/hal-01346433}

Submitted on 4 Aug 2016

HAL is a multi-disciplinary open access archive for the deposit and dissemination of scientific research documents, whether they are published or not. The documents may come from teaching and research institutions in France or abroad, or from public or private research centers.
L'archive ouverte pluridisciplinaire HAL, est destinée au dépôt et à la diffusion de documents scientifiques de niveau recherche, publiés ou non, émanant des établissements d'enseignement et de recherche français ou étrangers, des laboratoires publics ou privés. 


\title{
FULL PAPER
}

\section{A New Revised Desired Compensation Adaptive Control for Enhanced Tracking: Application to RA-PKMs}

\author{
Moussab Bennehar*a, Ahmed Chemori ${ }^{\mathrm{a}}$ and François Pierrot ${ }^{\mathrm{a}}$ \\ ${ }^{a}$ Department of Robotics, Laboratoire d'Informatique, de Robotique et de Microélectonique de Montpellier \\ (LIRMM), 161 rue Ada, 34090 Montpellier, France.
}

(v1.1 released March 2014)

\begin{abstract}
A new controller based on Desired Compensation Adaptation Law (DCAL) is proposed in this paper. The original DCAL control input can be split up into three main separate terms; an adaptive feedforward term, a Proportional-Derivative (PD) feedback term and a compensation term. Inspired from the fact that nonlinear time-varying feedback gains lead to improved performance, we propose in this work to revisit DCAL control scheme by replacing the constant feedback gains in the PD feedback term by nonlinear time-varying ones. The proposed nonlinear gains are automatically adjusted according to the variation of the tracking error yielding improved tracking performance. Besides, to cope with the internal forces issue that appears in the case of Redundantly Actuated Parallel Kinematic Manipulators (RA-PKMs), we propose to use a projection operator to reduce these forces. The projection operator, which is based on the kinematics of the manipulator, reduces the part of the control inputs responsible for internal forces. To demonstrate the relevance of the proposed control strategy, both standard DCAL and the proposed extended DCAL controller are experimentally implemented on a three degree of freedom (3-DOF) RAPKM called Dual-V. Based on the obtained results, it is shown that the proposed controller outperforms the original one in terms of tracking performance while reducing the control effort.
\end{abstract}

Keywords: Parallel manipulators; adaptive control; actuation redundancy; nonlinear control; real-time experiments.

\section{Introduction}

Parallel Kinematic Manipulators (PKMs) are mostly known for some impressive features and performances compared to classical serial ones [1]. Indeed, a comparison between these two types of manipulators shows that PKMs have higher load/weight ratio, higher stiffness, less sensitivity to sensors' errors and higher acceleration capabilities, among other enhanced features. These advantages, however, come with some drawbacks. From control point of view, the closed-chains constraints give raise to highly nonlinear dynamics, difficult to deal with. Moreover, uncertainties are abundant in such systems due to model simplifications, the wear of the components of the robot and the variations of the environment. Furthermore, their coupled dynamics and actuation redundancy in some mechanisms give rise to complex and challenging control issues.

Thanks to its design simplicity and efficiency, the Proportional-Integral-Derivative (PID) controller [2] has become the most used controller for industrial manipulators [3]. In such decentralized strategy, the control of the manipulator is reduced to controlling each axis separately by means of a PID feedback loop, regardless of any considerations about the coupled dynamics, the nonlinear nature of the system or its closed-chains structure. The nonlinear counterpart of the PID controller [4-8] has been introduced to provide enhanced performance and robustness compared to conventional

\footnotetext{
*Corresponding author. Email: bennehar@lirmm.fr
} 
linear one with constant gains. Its general principle is similar to the PID controller; however, the feedback gains are nonlinear functions mainly depending on the system's variables such as its state, the control input and/or other variables. In the literature, several nonlinear PID controllers have been proposed [6, 7]. Nevertheless, neither PID, nor nonlinear PID controllers can provide adequate performance when dealing with highly nonlinear time-varying systems such as PKMs, especially when operating at high accelerations. Consequently, more sophisticated control strategies taking the nonlinear dynamics of the system into account have to be investigated.

In several works, it was demonstrated that including some knowledge about the dynamics of the system in the control design procedure may lead to significant improvements [9, 10], especially in terms of tracking performance and energy consumption. Consequently, several controllers relying on the dynamics of the system were developed, such controllers are commonly called model-based controllers. The computed torque (CT) controller [11] is one of the most known strategies taking advantage of such technique. The CT controller is designed in such a way that all the nonlinearities of the manipulator are canceled, resulting in a group of second order decoupled linear systems. However, the CT controller suffers from the complexity of its structure and its sensitivity to measurement noise and model uncertainties. Consequently, various modifications have been proposed in the literature to cope with the former limitation culminating in the development of several controllers such as the augmented PD (APD) [12] and the PD+ [13] controllers. However, even with an accurate dynamic model, the issue of parameters' uncertainties cannot be solved by a model-based controller with constant model parameters. Indeed, the dynamics of a robot is known to be time-varying and is very likely to change according to the environment conditions (e.g. payload handling).

To overcome the shortcomings of model-based controllers with constant model parameters, modelbased adaptive control was developed [14]. The basic idea of model-based adaptive control is that an additional parameters estimation loop, based on the dynamics of the system and the tracking errors, is integrated in the control scheme. The adaptation law governing the evolution of the parameters' estimates is usually derived from a stability analysis of the closed-loop system. Although adaptive control has been extensively studied for serial manipulators, it did not gain the same success in PKMs. For instance, in [15], an adaptive joint-space controller was proposed to control a 6-DOF PKM. The proposed controller is based on Desired Compensation Adaptation Law (DCAL) control strategy [16] which was initially developed for serial manipulators. A task-space adaptive controller was proposed in [17] to control a 2-DOF planar RA-PKM. The adaptation law in this work is derived from the stability analysis of the closed-loop system based on Lyapunov theory and Barbalat's lemma. Experimental results shows that the proposed adaptive controller outperforms the APD in both low and high-speed motions. Unfortunately, the evolution of the estimated parameters was not provided to check whether they converge to their steady state values.

In this paper, we propose to revisit the standard DCAL controller [16] to improve its tracking performance. The control law of DCAL includes a PD feedback-loop with constant gains. We propose to replace these constant gains by nonlinear time-varying ones. The main motivation of such proposition is to improve the tracking performance of original DCAL. Furthermore, we propose to reduce the effect of the internal forces appearing exclusively in RA-PKMs through a projection operator. This last one is based on the kinematics of the manipulator and allows to significantly reduce the internal forces. This work is an extension of [18] where the enhanced DCAL controller with nonlinear feedback gains was proposed for the first time. The main contribution of this paper lies in the stability analysis of the closed-loop system under the proposed controller. The proposed controller as well as the original one are implemented in real-time on a planar 3-DOF RA-PKM prototype developed in our laboratory. The remainder of this paper is organized as follows; in Section 2, a brief background on DCAL control scheme is introduced. The proposed controller is then detailed and its stability analyzed in Section 3. In Section 4, we provide some necessary details about the PKM used in the real-time experiments. The obtained results are presented and discussed in Section 5 and, to finish, some conclusions and guidelines for future work are drawn in section 6 . 


\section{Background on DCAL Control Strategy}

\subsection{Dynamics of PKMs and its Linear-in-the-Parameters Reformulation}

In the general case, the number of degrees of freedom of a PKM is equal to the number of its actuators. However, in the particular case of PKMs with actuation redundancy (RA-PKMs), the number of degrees of freedom $m$ is less than the number of their actuators $n$ (i.e. $m<n$ ). Consider the general form of the joint space dynamics of a PKM expressed by [9]

$$
M(q) \ddot{q}+C(q, \dot{q}) \dot{q}+G(q)=\Gamma(t)
$$

where $M(q) \in \mathbb{R}^{n \times n}$ is the inertia matrix, $C(q, \dot{q}) \in \mathbb{R}^{n \times n}$ is the Coriolis and centrifugal forces matrix, $G(q) \in \mathbb{R}^{n}$ is the gravitational forces vector and $\Gamma(t) \in \mathbb{R}^{n}$ is the control inputs vector. $q, \dot{q}, \ddot{q} \in \mathbb{R}^{n}$ denote the actuated joints' position, velocity and acceleration vectors, respectively. It is worth to note that frictions' effects are not considered in the current work.

It has been reported in $[9,14,19]$ that the dynamic model of RA-PKMs and its matrices satisfy the following properties:

Property 1. $M(q)$ is symmetric, positive definite and satisfies the following inequality $\forall y \in \mathbb{R}^{n}$

$$
\underline{m}\|y\| \leq y^{T} M(q) y \leq \bar{m}(q)\|y\|,
$$

where $\underline{m}$ is a known positive constant, $\bar{m}(q)$ is a positive function and $\|$.$\| denotes the standard$ Euclidean norm.

Property 2. $\dot{M}(q)-2 C(q, \dot{q})$ is skew-symmetric.

Property 3. The dynamics of the manipulator (1) can be rewritten in a linear form with respect to the dynamic parameters. Indeed, according to [14], the dynamics (1) can be expressed as follows

$$
M(q) \ddot{q}+C(q, \dot{q}) \dot{q}+G(q)=Y(q, \dot{q}, \ddot{q}) \Theta
$$

where $Y(q, \dot{q}, \ddot{q}) \in \mathbb{R}^{n \times p}$ is called the regression matrix, which is a nonlinear function of the joint position, velocity and acceleration vectors. The vector $\Theta \in \mathbb{R}^{p}$ contains the different parameters to be estimated in real-time by the adaptation part of the controller. This vector of parameters can include separated or a combination of different physical (geometric and dynamic) parameters of the manipulator. Besides, it is worth to note that not all parameters should necessarily be considered for adaptation. In this particular case, the following, more general, reformulation is considered instead of $(2)$

$$
M(q) \ddot{q}+C(q, \dot{q}) \dot{q}+G(q)=Y_{n}(q, \dot{q}, \ddot{q}) \Theta_{n}+Y_{u}(q, \dot{q}, \ddot{q}) \Theta_{u}
$$

where $\Theta_{n} \in \mathbb{R}^{p n}$ is the set of known parameters, not considered for adaptation, and $\Theta_{u} \in \mathbb{R}^{p u}$ is the set of unknown or uncertain parameters to be estimated by the adaptation algorithm. $Y_{n}(q, \dot{q}, \dot{q}, \ddot{q}) \in$ $\mathbb{R}^{n \times p n}$ and $Y_{u}(q, \dot{q}, \dot{q}, \ddot{q}) \in \mathbb{R}^{n \times p u}$ are partial regression matrices with appropriate dimensions.

\subsection{DCAL control strategy and its advantages}

Let $q_{d}(t) \in \mathbb{R}^{n}$ denote the time evolution of the desired joint position trajectory that is twice differentiable such that

$$
q_{d}(t), \frac{d q_{d}(t)}{d t}, \frac{d^{2} q_{d}(t)}{d t^{2}} \in \mathcal{L}_{\infty}
$$


To quantify the control objective, let us define the joint tracking error $e(t) \in \mathbb{R}^{n}$ to be the difference between the desired position $q_{d}(t)$ and the actual one $q(t)$ as follows

$$
e(t)=q_{d}(t)-q(t)
$$

and define the combined tracking error $r(t) \in \mathbb{R}^{n}$ to be a combination of the joint position and velocity tracking errors as follows

$$
r(t)=\dot{e}(t)+\lambda e(t)
$$

where $\dot{e}(t)=\dot{q}_{d}(t)-\dot{q}(t)$ is the joint velocity tracking error and $\lambda$ is a positive control design scalar. The standard DCAL control law is formulated based on an adaptive exact compensation control scheme by replacing the measured trajectories in the dynamic-based compensation term by desired ones [16]. Indeed, in an exact compensation scheme, the complex nonlinear terms in the regression matrix are computed based on the measured joint positions and velocities which need to be available at each time-step. In addition to the time consuming nature of such algorithm, the velocities are usually not available and need to be estimated from the encoders' position measurements (e. g. numerical differentiation, low-pass filtering, etc.) [20]. It is however known that such estimation may amplify the noise and hurts the robustness of the closed-loop system. DCAL aims at solving such issue by replacing the measured trajectories by the desired ones. This is of a tremendous importance for real-time applications since the desired trajectories can be computed offline which can significantly reduce the computing time of the controller. Furthermore, the terms depending on desired trajectories are immune to measurement noise which enhances the robustness of the controller. The DCAL control law is given by [16]

$$
\Gamma(t)=Y\left(q_{d}, \dot{q}_{d}, \ddot{q}_{d}\right) \hat{\Theta}(t)+K_{p} e(t)+K_{v} r(t)+\sigma\|e(t)\|^{2} r(t)
$$

where $K_{p}, K_{v} \in \mathbb{R}^{n \times n}$ are positive-definite constant feedback gain matrices, $\sigma \in \mathbb{R}^{+}$a positive design parameter and $\hat{\Theta}(t) \in \mathbb{R}^{p}$ is an estimate of $\Theta$. The control law (7) can be seen as a combination of three distinct terms. The first term (i. e. $\left.Y\left(q_{d}, \dot{q}_{d}, \ddot{q}_{d}\right) \hat{\Theta}(t)\right)$ is the model-based part which is an adaptive feedforward responsible of the compensation of the nonlinearities of dynamics. The second term (i. e. $\left.K_{p} e(t)+K_{v} r(t)\right)$ is the feedback part which is responsible of compensating the residual tracking errors. The last term (i. e. $\left.\sigma\|e(t)\|^{2} r(t)\right)$ is an additional nonlinear feedback term responsible for compensating the errors that emerge from substituting the actual trajectories in the exact compensation scheme by desired ones. The parameters vector $\hat{\Theta} \in \mathbb{R}^{p}$ is an estimate of the nominal parameters vector $\Theta$. Its real-time evolution is governed by the following adaptation law

$$
\dot{\hat{\Theta}}(t)=K Y^{T}\left(q_{d}, \dot{q}_{d}, \ddot{q}_{d}\right) r(t)
$$

where $K \in \mathbb{R}^{p \times p}$ is a positive-definited adaptation gain matrix.

As it can be seen, the regression matrix in (8) which includes highly nonlinear terms is also based on the desired trajectories. Therefore, the adaptation process inherits the same advantages of DCAL cited above.

The block diagram illustrating DCAL control strategy is shown in Figure 1.

\section{Proposed Controller: An Extended DCAL with Nonlinear Feedback Gains}

\subsection{Background on Nonlinear Feedback Gains Theory}

The linear PID controller is the most used control strategy in industry. It is so known that it becomes a standard in industrial applications [3]. The basic control law of a linear PID controller takes the 


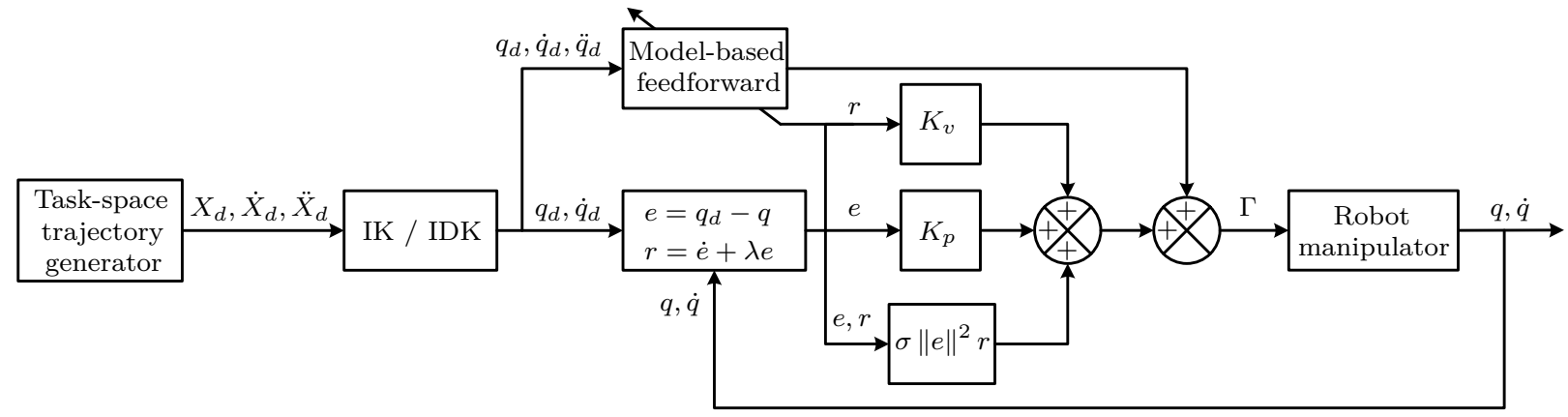

Figure 1. Block diagram of DCAL control scheme (IK: Inverse Kinematics, IDK: Inverse Differential Kinematics).

following form

$$
\Gamma(t)=K_{p} e(t)+K_{i} \int_{t_{0}}^{t} e(\tau) d \tau+K_{d} \dot{e}(t)
$$

where $K_{p}, K_{i}, K_{d} \in \mathbb{R}^{+}$are the feedback gains to be tuned to get the desired closed-loop performance. In the literature, it has been theoretically demonstrated that PID controllers guarantee asymptotic tracking and stability for linear systems. It is also reported that they provide a certain degree of robustness towards uncertainties and external disturbances, provided that the controller gains are chosen large enough. Nevertheless, these characteristics no longer hold for nonlinear systems.

To improve the performance of linear PID controllers and enhance their tracking and robustness capabilities, nonlinear PID controllers were proposed as an alternative solution. Indeed, nonlinear PID controllers share the same simple design structure with their linear counterparts. However, instead of taking constant feedback gains, nonlinear PID controllers use time-varying gains depending on information about the system such as its state and the control input. Thus, a typical nonlinear PID controller is any controller with the following form

$$
\Gamma(t)=K_{p}(.) e(t)+K_{i}(.) \int_{t_{0}}^{t} e(\tau) d \tau+K_{d}(.) \dot{e}(t)
$$

where the feedback gains $K_{p}(),. K_{i}(),. K_{d}($.$) , in this case, are nonlinear functions depending on dif-$ ferent variables of the system such as the state, the control inputs or other variables. The fact that nonlinear PID controllers use nonlinear time-varying gains, instead of constant ones, enables different choices of the basic functions used for these gains. Indeed, various controllers with nonlinear feedback gains have been proposed in the literature [4-8]. Every controller has a specific form chosen according to the control objective.

Attracted by the advantages of nonlinear feedback gains, several works proposed not only to use nonlinear PID controllers, but even to revisit other more sophisticated model-based controllers using constant gains in their corresponding feedback loops [21-23]. Since model-based adaptive controllers share a similar structure with non-adaptive ones, it would be interesting to revisit model-based adaptive controllers to include nonlinear time-varying gains instead of constant ones as well.

\section{2. $\quad$ Extended DCAL with nonlinear feedback gains}

As described earlier, the control law of DCAL (7) can be decomposed into three terms. A nonlinear adaptive feedforward term, a PD feedback term and a compensation term. Roughly speaking, the main idea of our proposed contribution is to replace the constant feedback gains in DCAL by nonlinear time-varying ones in order to enhance the performance of the controller. Hence, the proposed 
enhanced controller can be expressed as follows

$$
\Gamma(t)=Y\left(q_{d}, \dot{q}_{d}, \ddot{q}_{d}\right) \hat{\Theta}(t)+K_{p}(.) e(t)+K_{v}(.) r(t)+\sigma\|e(t)\|^{2} r(t)
$$

where $K_{p}($.$) and K_{v}($.$) are nonlinear time-varying feedback gain matrices to be adequately designed.$ Since we are interested in improving the trajectory tracking performance of PKMs, one interesting choice for the time-evolution of the nonlinear gains would be as follows. When the tracking error is large, an amplified control action is required to reject this large tracking error as fast as possible. As the tracking error starts to decrease, reduce the control action to avoid oscillations and large overshoots. We also need for the control scheme to have a structure simple enough for real-time implementation.

The nonlinear gains in the case of PKMs are usually taken diagonal, which means that no coupling between the actuators is considered. Hence, $K_{p}($.$) and K_{v}($.$) in (11) can be expressed as follows$

$$
K_{p}(.)=\operatorname{diag}\left(k_{p_{1}}(.), k_{p_{2}}(.), \ldots, k_{p_{n}}(.)\right), \quad K_{v}(.)=\operatorname{diag}\left(k_{v_{1}}(.), k_{v_{2}}(.), \ldots, k_{v_{n}}(.)\right)
$$

Similar to Shang [21-23], we propose to use the nonlinear gains proposed in [4] which satisfy our requirements. The $k_{p_{i}}($.$) and k_{v_{i}}($.$) elements in (12) in this case are expressed as$

$$
\begin{aligned}
& k_{p_{i}}\left(e_{i}, \alpha_{p}, \delta_{p}\right)= \begin{cases}k_{p_{0}}\left|e_{i}(t)\right|^{\alpha_{p}-1}, & \left|e_{i}(t)\right|>\delta_{p} \\
k_{p_{0}} \delta_{p}^{\alpha_{p}-1}, & \left|e_{i}(t)\right| \leq \delta_{p}\end{cases} \\
& k_{v_{i}}\left(r_{i}, \alpha_{v}, \delta_{v}\right)= \begin{cases}k_{v_{0}}|r(t)|^{\alpha_{v}-1}, & \left|r_{i}(t)\right|>\delta_{v} \\
k_{v_{0}} \delta_{v}^{\alpha_{v}-1}, & \left|r_{i}(t)\right| \leq \delta_{v}\end{cases}
\end{aligned}
$$

where $k_{p_{0}}, k_{v_{0}}, \alpha_{p}, \delta_{p}, \alpha_{v}, \delta_{v}$ are positive design parameters to be tuned to get the desired performance. If the control design parameters $\alpha_{p}$ and $\alpha_{v}$ are chosen such that $\alpha_{p}>1$ and $\alpha_{v}>1$, then the following bounds hold globally for the nonlinear gains $K_{p}($.$) and K_{v}($.$) given by (13a) and (13b), respectively:$

$$
\begin{aligned}
& 0<K_{p_{m}} \triangleq k_{p_{0}} \delta_{p}^{\alpha_{p}-1} \mathbb{I}_{n} \leq K_{p}(.) \leq k_{p_{0}}\left(\delta_{p}^{\alpha_{p}-1}+\|e\|_{\infty}^{\alpha_{p}-1}\right) \mathbb{I}_{n} \triangleq K_{p_{M}} \\
& 0<K_{v_{m}} \triangleq k_{v_{0}} \delta_{v}^{\alpha_{v}-1} \mathbb{I}_{n} \leq K_{v}(.) \leq k_{v_{0}}\left(\delta_{v}^{\alpha_{v}-1}+\|r\|_{\infty}^{\alpha_{v}-1}\right) \mathbb{I}_{n} \triangleq K_{v_{M}}
\end{aligned}
$$

with $\mathbb{I}_{n}$ being a $n \times n$ identity matrix and $\|\cdot\|_{\infty}$ denotes the infinity-norm.

An illustration of the time evolution of the proposed nonlinear gains is shown in Figure 2.

Regarding the evolution of the estimated parameters, the same adaptation rule (8) as the original controller is used. Therefore, the proposed controller inherits the advantages from both DCAL and nonlinear PD feedback control.

\subsection{Stability analysis of the proposed extended DCAL controller}

Theorem 1. The joint position and velocity tracking errors $(e(t)$ and $\dot{e}(t)$, respectively) of a mechanical manipulator whose dynamics is governed by (1) under the controller (11) goes to zero as time goes to infinity, provided that the control design parameters $\alpha_{p}$ and $\alpha_{v}$ are chosen such that $\alpha_{p}>1$ and $\alpha_{v}>1$ and that the lower bounds $K_{p_{m}}$ and $K_{v_{m}}$ in (14) and (15), respectively, are chosen large enough.

Proof. To analyze the stability of the PKM in closed-lopp with the proposed extended DCAL con- 
troller, let us first rewrite its dynamics (1) in terms of $e(t), \dot{e}(t)$ and $r(t)$ as follows

$$
M(q) \dot{r}=M(q)\left(\ddot{q}_{d}+\lambda \dot{e}\right)+C(q, \dot{q})\left(\dot{q}_{d}+\lambda e\right)-C(q, \dot{q}) r+G(q)-\Gamma
$$

By adding and subtracting $Y\left(q_{d}, \dot{q}_{d}, \ddot{q}_{d}\right) \Theta$ to (16), we obtain

$$
M(q) \dot{r}=-C(q, \dot{q}) r+\tilde{Y}(.) \Theta+Y\left(q_{d}, \dot{q}_{d}, \ddot{q}_{d}\right) \Theta-\Gamma
$$

where $\tilde{Y}($.$) is the error in the dynamics emerging from using the desired trajectories instead of the$ measured ones. It given by

$$
\tilde{Y}(.) \Theta \triangleq M(q)\left(\ddot{q}_{d}+\lambda \dot{e}\right)+C(q, \dot{q})\left(\dot{q}_{d}+\lambda e\right)+G(q)-Y\left(q_{d}, \dot{q}_{d}, \ddot{q}_{d}\right) \Theta
$$

Applying the control input of the proposed extended DCAL controller (11) to the error dynamics in (17) results in

$$
M(q) \dot{r}=-C(q, \dot{q}) r-K_{p}(.) e-K_{v}(.) r-\sigma\|e\|^{2} r+\tilde{Y}(.) \Theta+Y_{d}(.) \tilde{\Theta}(t)
$$

with $\tilde{\Theta}(t) \triangleq \Theta-\hat{\Theta}(t)$ is the estimation error vector and $Y_{d} \triangleq Y\left(q_{d}, \dot{q}_{d}, \ddot{q}_{d}\right)$.

For the stability analysis of the system's error dynamics given by (19), consider the following Lyapunov candidate

$$
V=\frac{1}{2} r^{T} M(q) r+\frac{1}{2} K_{p_{m}} e^{T} e+\frac{1}{2} \tilde{\Theta}^{T} K^{-1} \tilde{\Theta}
$$

whose first time-derivative leads to

$$
\dot{V}=\frac{1}{2} r^{T} \dot{M}(q) r+r^{T} M(q) \dot{r}+K_{p_{m}} e^{T} \dot{e}+\tilde{\Theta}^{T} K^{-1} \dot{\tilde{\Theta}}
$$

Using the skew-symmetric property of $\dot{M}(q)-2 C(q, \dot{q})$ and the adaptation law $(8), \dot{V}$ can be rewritten as follows

$$
\dot{V}=K_{p_{m}} e^{T} \dot{e}-r^{T} K_{p}(.) e-r^{T} K_{v}(.) r-\sigma\|e\|^{2}\|r\|^{2}+r^{T} \tilde{Y}(.) \Theta
$$

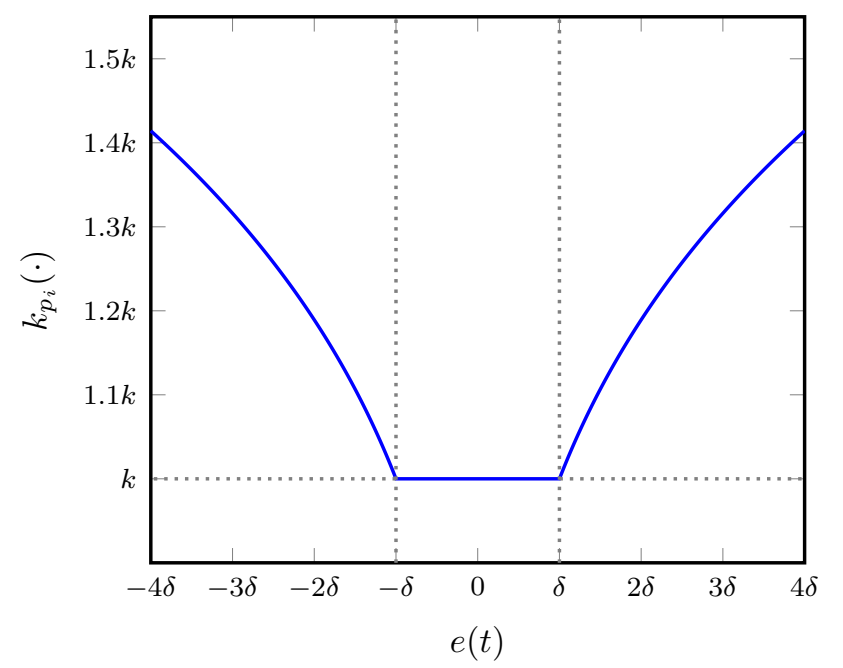

Figure 2. Evolution of the proposed nonlinear gains versus the tracking error 
Following the same reasoning in [16], $\tilde{Y}(.) \Theta$ can be upper-bounded as follows

$$
\|\tilde{Y}(.) \Theta\| \leq \zeta_{1}\|e\|+\zeta_{2}\|e\|^{2}+\zeta_{3}\|r\|+\zeta_{4}\|r\|\|e\|
$$

where $\zeta_{1}, \zeta_{2}, \zeta_{3}$ and $\zeta_{4}$ are positive bounding constants. If we consider the upper bound on $\|\tilde{Y}(.) \Theta\|$ together with the lower bounds on $K_{p}($.$) and K_{v}(),. \dot{V}$ can be upper-bounded as follows

$$
\dot{V} \leq-\lambda K_{p_{m}}\|e\|^{2}-K_{v_{m}}\|r\|^{2}-\sigma\|e\|^{2}\|r\|^{2}+\zeta_{1}\|e\|\|r\|+\zeta_{2}\|e\|^{2}\|r\|+\zeta_{3}\|r\|^{2}+\zeta_{4}\|r\|^{2}\|e\|
$$

Rearranging the terms of (24), it can be rewritten as

$$
\begin{aligned}
\dot{V} \leq- & \lambda K_{p_{m}}\|e\|^{2}-K_{v_{m}}\|r\|^{2}-\sigma\|e\|^{2}\|r\|^{2}+\zeta_{1}\|e\|\|r\|+\zeta_{2}\|e\|^{2}\left[\frac{1}{2}-\|r\|\right]^{2} \\
& +\zeta_{4}\|r\|^{2}\left[\frac{1}{2}-\|e\|^{2}+\left(\zeta_{2}+\zeta_{4}\right)\|e\|^{2}\|r\|^{2}+\frac{\zeta_{2}}{4}\|e\|^{2}+\left(\zeta_{3}+\frac{\zeta_{4}}{4}\right)\|r\|^{2}\right.
\end{aligned}
$$

Then, by collecting common terms of (25), we obtain

$$
\begin{aligned}
\dot{V} \leq- & \left(\lambda K_{p_{m}}-\frac{\zeta_{2}}{4}\right)\|e\|^{2}-\left(K_{v_{m}}-\zeta_{3}-\frac{\zeta_{4}}{4}\right)\|r\|^{2}+\zeta_{1}\|e\|\|r\| \\
& -\zeta_{2}\|e\|^{2}\left[\frac{1}{2}-\|r\|\right]^{2}-\zeta_{4}\|r\|^{2}\left[\frac{1}{2}-\| e\right]^{2}-\left(\sigma-\zeta_{2}-\zeta_{4}\right)\|e\|^{2}\|r\|^{2}
\end{aligned}
$$

If the control gain $\sigma$ is chosen such that $\left(\sigma-\zeta_{2}-\zeta_{4}\right)>0$, then, $\dot{V}$ can be upper-bounded with the following new bound

$$
\dot{V} \leq-\left(\lambda K_{p_{m}}-\frac{\zeta_{2}}{4}\right)\|e\|^{2}-\left(K_{v_{m}}-\zeta_{3}-\frac{\zeta_{4}}{4}\right)\|r\|^{2}+\zeta_{1}\|e\|\|r\|
$$

Then, considering the fact that

$$
\|e\|\|r\| \leq \frac{\|e\|^{2}}{2}+\frac{\|r\|^{2}}{2}
$$

leads to the new upper-bound on $\dot{V}$ as follows

$$
\dot{V} \leq-\left(\lambda K_{p_{m}}-\frac{\zeta_{2}}{4}-\frac{\zeta_{1}}{2}\right)\|e\|^{2}-\left(K_{v_{m}}-\zeta_{3}-\frac{\zeta_{4}}{4}-\frac{\zeta_{1}}{2}\right)\|r\|^{2}
$$

Consequently, if the lower bounds $K_{p_{m}}$ and $K_{v_{m}}$ are tuned such that

$$
\begin{array}{r}
\lambda K_{p_{m}}-\frac{\zeta_{2}}{4}-\frac{\zeta_{1}}{2}>0 \\
K_{v_{m}}-\zeta_{3}-\frac{\zeta_{4}}{4}-\frac{\zeta_{1}}{2}>0
\end{array}
$$

then, $\dot{V}$ would be negative semi-definite. Considering the bound inequalities of $K_{p}($.$) and K_{v}($.$) in$ 
(14) and (15), respectively, we can notice that if the gains parameters are chosen such that

$$
\begin{aligned}
& k_{p_{0}} \delta_{p}^{\alpha_{p}-1}>\frac{\zeta_{2}}{4 \lambda}+\frac{\zeta_{1}}{2 \lambda} \\
& k_{v_{0}} \delta_{v}^{\alpha_{v}-1}>\zeta_{3}+\frac{\zeta_{4}}{4}+\frac{\zeta_{1}}{2}
\end{aligned}
$$

then, the inequalities (32) and (33) are satisfied. Consequently, $\dot{V}$ is negative semi-definite, therefore, $V$ is upper bounded. Then, based on the definition of $V$, we conclude that $e, r, \dot{e}$ and $\tilde{\Theta}$ are all bounded. Eq. (19) can be used to show that $\dot{r}(t), \ddot{q}(t)$ and, consequently, $\ddot{V}$ are all bounded. Given the fact that the inertia matrix $M(q)$ is lower bounded, it can be noticed that $V$ in (20) is lower bounded as well. Since $V$ is lower bounded, $\dot{V}$ is negative semi-definite and $\ddot{V}$ is bounded (i.e. $\dot{V}$ is uniformally continuous), Barbalat's Lemma $[24,25]$ can be invoked to state that

$$
\lim _{t \rightarrow \infty} \dot{V}=0
$$

Therefore, from the definition of $\dot{V}$ and $r$, we conclude that

$$
\begin{aligned}
& \lim _{t \rightarrow \infty} e=0 \\
& \lim _{t \rightarrow \infty} \dot{e}=0
\end{aligned}
$$

which concludes the proof. Notice that the stability analysis does not say anything about the estimated parameters except that they remain bounded.

\subsection{Projection method for reducing internal forces}

For the particular case of PKMs with actuation redundancy, the control inputs, if not carefully designed, may generate internal forces [26]. This issue can be caused by geometric uncertainties, the finite resolution of the encoders and the use of decentralized control. The internal forces can be used for secondary tasks such as modulating the stiffness and avoiding backlash. However, if these forces are not allocated for secondary tasks, they should be attenuated as much as possible.

In [26], it was demonstrated that the internal forces are those control inputs in the null-space of the transpose of the inverse Jacobian matrix $J_{m}(q) \in \mathbb{R}^{n \times m}$. Therefore, to get rid of the internal forces, we need to eliminate all the control inputs in the null-space of $J_{m}^{T}$ which can be achieved using the following projection operator [26]

$$
R_{j_{m}^{T}} \triangleq I_{n \times n}-N_{j_{m}^{T}}
$$

where $I_{n \times n}$ is a $(n \times n)$ identity matrix and $N_{j_{m}^{T}} \in \mathbb{R}^{n \times n}$ is the null-space projector. Hence, the regularized control input to be applied to the manipulator is given by

$$
\Gamma^{*}=R_{j_{m}^{T}} \Gamma_{\text {input }}
$$

where $\Gamma_{\text {input }}$ is the control input computed by any control scheme (e. g. PD, DCAL, extended DCAL, etc.).

The block diagram illustrating the proposed extended DCAL controller with the projection operator for the case of RA-PKMs is shown in Figure 3. 
Table 1. Main parameters of Dual-V robot.

\begin{tabular}{cccccc}
\hline Parameter & Description & Value & Parameter & Description & Value \\
\hline$l_{c r}$ & Cranks' length & $0.28 \mathrm{~m}$ & $l_{c o}$ & Coupler' length & $0.28 \mathrm{~m}$ \\
$m_{c r}$ & Crank's mass & $1.169 \mathrm{~kg}$ & $m_{c o}$ & Coupler' mass & $0.606 \mathrm{~kg}$ \\
$I_{c r}$ & Cranks' inertia & $0.01297 \mathrm{kgm}^{2}$ & $I_{c o}$ & Couplers' inertia & $0.00642 \mathrm{kgm}^{2}$ \\
$l_{p}$ & Platform's length & $0.22 \mathrm{~m}$ & $I_{c m}$ & Counter-masses inertia & $0.02685 \mathrm{kgm}^{2}$ \\
$m_{p}$ & Platform's mass & $0.899 \mathrm{~kg}$ & $m_{c m}$ & Counter-masses mass & $7.983 \mathrm{~kg}^{2}$ \\
$I_{p}$ & Platform's inertia & $0.22 \mathrm{kgm}^{2}$ & $I_{a}$ & Actuator's inertia & $0.0041 \mathrm{kgm}^{2}$ \\
\hline
\end{tabular}

\section{Description of Dual-V: a 3-DOF Planar RA-PKM}

The Dual-V robot [27] is a 3-DOF planar RA-PKM developed within a collaboration between LIRMM laboratory (France) and the University of Twente (Netherlands). It belongs to the 4-RRR family in which, every $\underline{R R R}$ chain is composed of an actuator, a crank and a coupler (cf. Figure 4). The joint position vector of Dual-V is denoted by $q \triangleq\left[q_{1}, q_{2}, q_{3}, q_{4}\right]^{T} \in \mathbb{R}^{4}$. The manipulator's links are made with aluminum and the arrangement of the four $\underline{R} R R$ chains allows one rotation and two translations of its moving platform. Hence, the position of its moving platform is described by $X \triangleq[x, y, \alpha]^{T} \in \mathbb{R}^{3}$. Table 1 summarizes the main dynamic and geometric parameters of Dual- $\mathrm{V}$ robot.

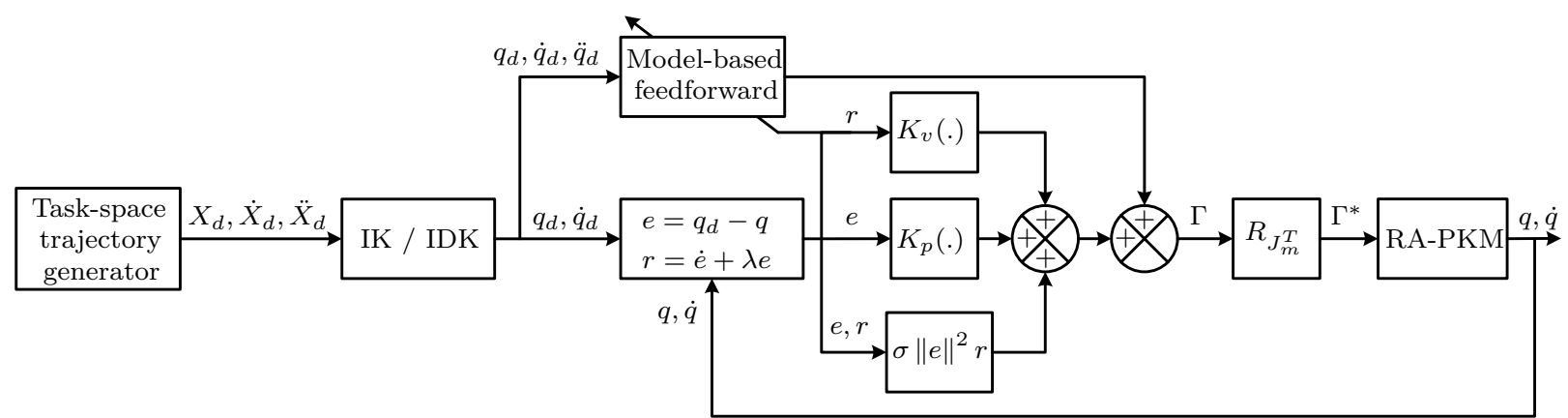

Figure 3. Block diagram of the proposed extended DCAL controller with projection operator.

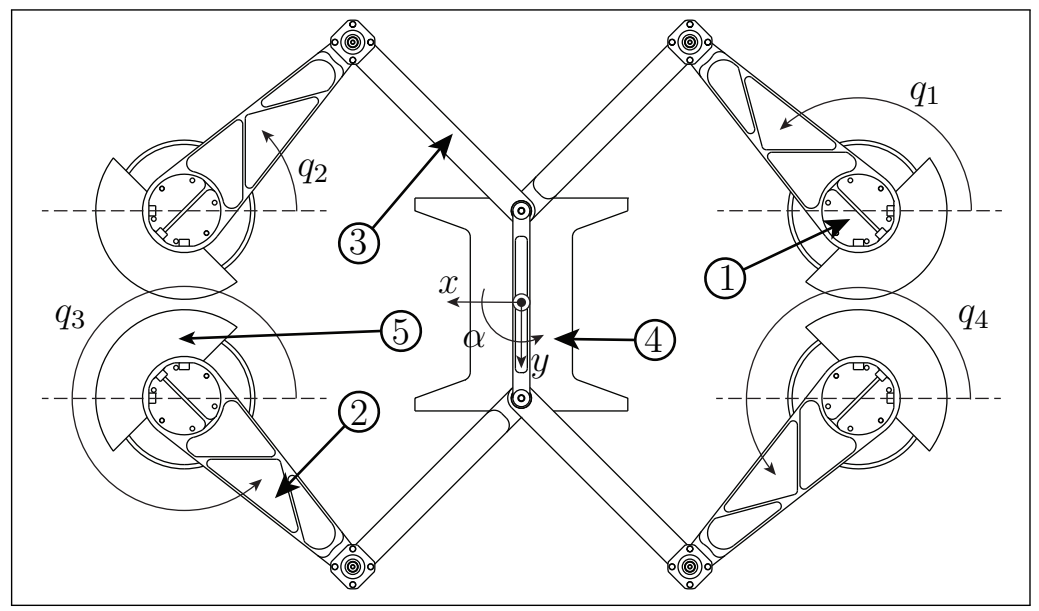

Figure 4. Schematic design of the Dual-V robot. (1) actuator, (2) arm (crank), (3) forearm (coupler), (4) moving platform and (5) counter-mass. 


\subsection{Dynamic modeling of Dual- $V$ robot}

The full Cartesian space inverse dynamic model, required for DCAL and extended DCAL controllers can be expressed as follows [27]:

$$
J_{m}^{T+} M_{T P} \ddot{X}+M_{T A} \ddot{q}+\Gamma_{c}(t)=\Gamma(t)
$$

where

- $J_{m}(q, X) \in \mathbb{R}^{4 \times 3}$ denotes the inverse Jacobian matrix linking the moving platform's velocities to those of the actuators (such that $\dot{q}=J_{m} \dot{X}$ ).

- $(.)^{+}$denotes the pseudoinverse of (.).

- $M_{T P} \triangleq \operatorname{diag}\left(m_{t p}, m_{t p}, I_{t p}\right) \in \mathbb{R}^{3 \times 3}$ is a diagonal constant matrix gathering the inertial parameters of the platform in addition to half of the total mass of the couplers.

- $\ddot{X} \triangleq[\ddot{x}, \ddot{y}, \ddot{\alpha}]^{T} \in \mathbb{R}^{3}$ and $\ddot{q} \in \mathbb{R}^{4}$ are the Cartesian and joint accelerations, respectively.

- $M_{T A} \in \mathbb{R}^{4 \times 4}$ is a diagonal constant matrix gathering the inertial parameters of the arms, the counter masses and the half of the total mass of the forearms.

- $\Gamma_{c}$ is an compensation term added for compensating the nonlinearities resulting from the simplifying hypotheses [27].

- $\Gamma \in \mathbb{R}^{4}$ is the input torque vector.

The dynamics of Dual-V robot (39) can be put into the standard joint space form, using adequate kinematic relationships, as follows:

$$
\left(M_{T A}+J_{m}^{T+} M_{T P} J_{m}^{+}\right) \ddot{q}-\left(J_{m}^{T+} M_{T P} J_{m}^{+} \dot{J}_{m} J_{m}\right) \dot{q}+\Gamma_{c}(t)=\Gamma(t)
$$

where $\dot{q} \in \mathbb{R}^{4}$ is the joint velocities vector.

For more details on the dynamic modeling of Dual- $\mathrm{V}$ robot, the reader is referred to [27] where more details about the dynamic modelling and additional information about Dual-V tobot are provided.

\subsection{Description of the experimental testbed}

The experimental setup used for the real-time validation of the proposed control scheme is shown in Figure 5. As it can be seen, the experimental platform is a planar 3-DOF RA-PKM mounted on an aluminum frame. The manipulator is equipped with four direct drive actuators (rotatory motors) provided by $E T E L^{\circledR}$. Each actuator is capable of delivering up to $127 \mathrm{Nm}$ of peak torque and $22 \mathrm{Nm}$ of nominal continuous torque. The maximum rotation speed of each actuator is about $550 \mathrm{rpm}$ and its maximum acceleration is $10000 \mathrm{rad} / \mathrm{s}^{2}$. The actuators are equipped with high-precision non-contact

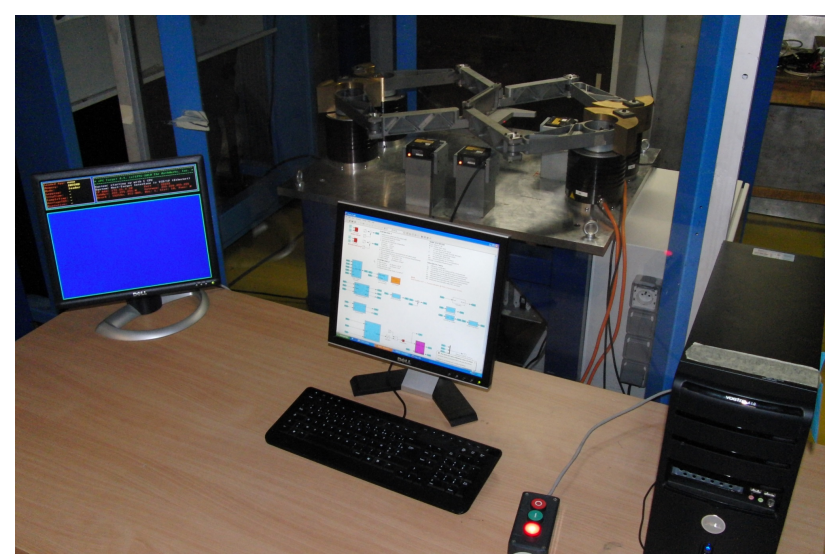

Figure 5. View of the experimental setup of Dual-V robot 
incremental optical encoders with 5000 lines of the grating disk.

The control algorithms are implemented using Matlab and Simulink, both from Mathworks ${ }^{\circledR}$, Inc. with the Real-Time Workshop toolbox for the $\mathrm{C}$ code generation. The generated code is then uploaded to the industrial target PC cadenced at a frequency of $10 \mathrm{kHz}$ and running xPC Target in real-time. For comparison purposes, both the standard DCAL and the extended DCAL control schemes are implemented. Also, to cope with the issue of actuation redundancy, both control laws use the projection operator (37)-(38).

\section{Real-time Experiments and Obtained Results}

In what follows, a detailed description of the implementation of DCAL and the extended DCAL controllers on our experimental platform is provided.

\subsection{Reference trajectories generation}

In our experimental scenarios, the reference trajectories to be tracked by the robot belong to the class of point-to-point trajectories [28]. The end-effector of the robot has to move from an initial Cartesian position denoted by $X_{0}$ to a final one denoted by $X_{f}$ with zero velocity and acceleration. Once the end-effector reaches the final position $X_{f}$, the next target position is fed to the trajectory generator. Hence, the previous final position becomes the new $X_{0}$, while the new target position becomes $X_{f}$, and so on.

The evolution of the trajectory between $X_{0}$ and $X_{f}$ with respect to time is governed by the following equation

$$
\begin{aligned}
X_{d}(t) & =X_{0}+D \nu(t) \\
\dot{X}_{d}(t) & =D \dot{\nu}(t) \\
\ddot{X}_{d}(t) & =D \ddot{\nu}(t)
\end{aligned}
$$

where $D \triangleq X_{f}-X_{0}$ is the distance to be run through and $\nu(t)$ is a fifth degree polynomial given by

$$
\nu(t)=10\left(\frac{t-t_{0}}{T}\right)^{3}-15\left(\frac{t-t_{0}}{T}\right)^{4}+6\left(\frac{t-t_{0}}{T}\right)^{5}, \quad \nu\left(t_{0}\right)=0, \nu\left(t_{0}+T\right)=1
$$

where $t_{0}$ is the initial time of the trajectory and $T$ its duration that has to be chosen by the user with careful consideration of the manipulator's capacities.

To compute the joint trajectory $q_{d}(t)$ corresponding to the Cartesian trajectory $X_{d}(t)$, inverse

Table 2. Sequence of points for the interpolated trajectory used in experiments

\begin{tabular}{ccccc}
\hline Point & $\mathbf{x}[\mathbf{m m}]$ & $\mathbf{y}[\mathbf{m m}]$ & $\alpha[\mathbf{d e g}]$ & $\mathbf{T}[\mathbf{s}]$ \\
\hline$X_{0}$ & 0 & 0 & 0 & \\
$X_{1}$ & 40 & 100 & 0 & 0.25 \\
$X_{2}$ & 0 & 0 & 0 & 0.25 \\
$X_{3}$ & -40 & -100 & 0 & 0.25 \\
$X_{4}$ & 0 & 0 & 0 & 0.25 \\
$X_{5}$ & 0 & 0 & 10 & 0.25 \\
$X_{6}$ & 0 & 0 & 0 & 0.25 \\
$X_{7}$ & 0 & 0 & -10 & 0.25 \\
$X_{8}$ & 0 & 0 & 0 & 0.25 \\
\hline
\end{tabular}


kinematics (IK) and invese differential kinematics (IDK) are used as follows:

$$
\begin{aligned}
& q_{d}(t)=I K\left(X_{d}(t)\right) \\
& \dot{q}_{d}(t)=J_{m} \dot{X}_{d}(t) \\
& \ddot{q}_{d}(t)=J_{m} \ddot{X}_{d}(t)+\dot{J}_{m} \dot{X}_{d}(t)
\end{aligned}
$$

In our experimental case study, a trajectory consisting of 8 poses of the moving platform (summarized in table 2) of the robot is considered.

\subsection{Experimental scenarios and some design issues}

For the real-time experiments we propose to validate two scenarios. The first one is the nominal case while in the second one, an additional weight is added on top of the moving platform. Therefore, the mostly effected parameters of the manipulator are those of the moving platform. Consequently we consider in our control algorithm that only the parameters of the moving platform are unknown; namely its mass and rotational inertia around its center of mass (CoM). This means that the reformulation of the dynamics of Dual-V (39) takes the form of (3). Hence, the dynamics of DUAL-V is rewritten as follows

$$
\left(M_{T A}+J_{m}^{T+} M_{T P} J_{m}^{+}\right) \ddot{q}-\left(J_{m}^{T+} M_{T P} J_{m}^{+} \dot{J}_{m} J_{m}\right) \dot{q}+\Gamma_{C}(t)=Y_{u}(q, \ddot{X}) \Theta_{u}+M_{T A} \ddot{q}+\Gamma_{C}(t)
$$

That is, only the dynamics of the moving platform is reformulated. The regression matrix $Y_{u} \in$ $\mathbb{R}^{4 \times 2}$ is given by

$$
Y_{u}=\left(J_{m}^{T+}\right)\left[\begin{array}{cc}
\ddot{x} & 0 \\
\ddot{y} & 0 \\
0 & \ddot{\alpha}
\end{array}\right]
$$

where $\ddot{x}, \ddot{y}, \ddot{\alpha}$ are the components of the Cartesian acceleration vector. The reduced estimated parameters vector $\Theta_{u} \in \mathbb{R}^{2}$ contains only the mass and the rotational inertia of the moving platform in addition to the four half-masses corresponding to the couplers, that is

$$
\Theta_{u}=\left[\begin{array}{c}
m_{t p} \\
I_{t p}
\end{array}\right]
$$

Therefore, the control law to be applied to the manipulator is given by

$$
\Gamma^{*}(t)=R_{j_{m}^{T}}\left[\Gamma_{f b}+Y_{u}\left(q_{d}, \ddot{X}_{d}\right) \hat{\Theta}_{u}(t)+M_{T A} \ddot{q}_{d}+\Gamma_{C_{d}}(t)+\sigma\|e(t)\|^{2} r(t)\right]
$$

where $\Gamma_{f b}$ is the feedback control term (PD with constant gains in the case of DCAL or nonlinear PD with time-varying gains in the case of EDCAL) and $M_{T A} \ddot{q}_{d}+\Gamma_{C_{d}}(t)$ is the feedforward compensation term evaluated using desired trajectories. $\hat{\Theta}_{u}(t)$ is the estimation of $\Theta_{u}$, governed by the following adaptation law

$$
\dot{\hat{\Theta}}_{u}(t)=K Y_{u}^{T}\left(q_{d}, \ddot{X}_{d}\right) r(t)
$$

\subsection{Tuning of the nonlinear feedback gains}

The tuning of the feedback gains of any controller is a crucial and important task that requires a careful attention. Indeed, it is well known that different combinations of PD gains leads to drastically 
different closed-loop behavior. Even though there exists a variety of theoretical tuning methods, the intuitive experimental trial and error technique remains the most used one. This is mainly due to the fact that the theoretical methods consider an idealized simplified dynamic model that does not exactly match the physical system. Consequently, theoretically computed gains may even lead to instability of the closed-loop system. For the case of our platform, the obtained constant gains that lead to desired performance, obtained using trial and error, are as follows: $K_{p}=3500$ and $K_{v}=25$.

For the nonlinear gains, the following tuning algorithm was used to obtain the control parameters leading to the desired performance

(1) Set $\alpha_{p}=1, \alpha_{v}=1$ and tune $\sigma_{p}$ and $\sigma_{v}$ as regular constant gains (by trial and error for instance).

(2) Measure the maximum position error $e_{\max }$ and the maximum combined error $r_{\max }$ under this configuration.

(3) Select $\delta_{p}=e_{\max } / 2$ and $\delta_{v}=r_{\max } / 2$.

(4) Increase progressively both values of $\alpha_{p}$ and $\alpha_{v}$ as well as the values of $\sigma_{p}$ and $\sigma_{v}$ until the desired performance is achieved.

Based on the above tuning algorithm, the following controller parameters are obtained: $k_{p_{0}}=$ $44000, \alpha_{p}=1.45, \delta_{p}=2 \times 10^{-3}, k_{v_{0}}=75, \alpha_{v}=1.3$ and $\delta_{v}=0.02$.

The diagonal adaptation gain matrix $K$ is progressively increased until fast enough convergence of the parameters is obtained. High oscillations in the estimated parameters due to high adaptation gains should be avoided since it can lead to instability. The adaptation gain matrix that lead to desired was $K=\operatorname{diag}\left(1,2 \times 10^{-2}\right)$. The estimated parameters were initialized to zero, i.e. $\hat{\Theta}\left(t_{0}\right)=$ $[0,0]^{T}$.

\subsection{Performance evaluation criteria}

To fairly evaluate and compare the performance of both controllers, we need to define some performance criteria. One of the main objective behind the control of mechanical manipulators is to track the reference trajectories as accurate as possible. Hence, it is quite appropriate to introduce some criteria based on the tracking errors to quantify this degree of accuracy. Let us introduce the following Root Mean Square (RMS) based criteria for the translational motion of the moving platform

$$
\operatorname{RMSE}_{x y}=\frac{1}{N} \sqrt{\sum_{i=1}^{N}\left[\left(\hat{x}(i)-x_{d}(i)\right)^{2}+\left(\hat{y}(i)-y_{d}(i)\right)^{2}\right]}
$$

where $N$ is the number of samples, $\hat{x}$ and $\hat{y}$ denote measured values of $x$ and $y$, respectively. Similarly, a performance criteria for the rotational movement of the moving platform is also introduced as follows

$$
\operatorname{RMSE}_{\alpha}=\frac{1}{N} \sqrt{\sum_{i=1}^{N}\left(\hat{\alpha}(i)-\alpha_{d}(i)\right)^{2}}
$$

where $\hat{\theta}$ is the measured value of $\theta$. It is worth to note that our experimental prototype is not equipped with external sensors to measure the Cartesian positions of the moving platform. Hence, they are obtained by solving forward kinematics based on the measured joint positions.

\subsection{Experimental results}

In the following we will provide details about the obtained experimental results. We perform two scenarios to show the effectiveness of the proposed control scheme and its adaptive algorithm. 


\subsubsection{Scenario 1: nominal case}

In this scenario, no payload was added to the moving platform of DUAL-V robot. However, we suppose that its dynamic parameters are unknown. A comparison between the Cartesian tracking errors of both controllers is illustrated in Figure 6. For clarity of the view, Figure 7 shows the same Cartesian tracking errors being zoomed around the interval $[11,14]$ seconds. It can be clearly seen that the proposed extended DCAL controller provides better results than the standard DCAL. Thanks to their time-varying property, the nonlinear feedback gains adjust themselves depending on to the magnitudes of the tracking errors. This allows to quickly reject large errors while avoiding large overshoots. The above RMS-based criteria are used to evaluate the enhancement brought by the proposed controller. The obtained results are summarized in Table 3 from which we can see that the tracking errors are reduced by up to $13 \%$.

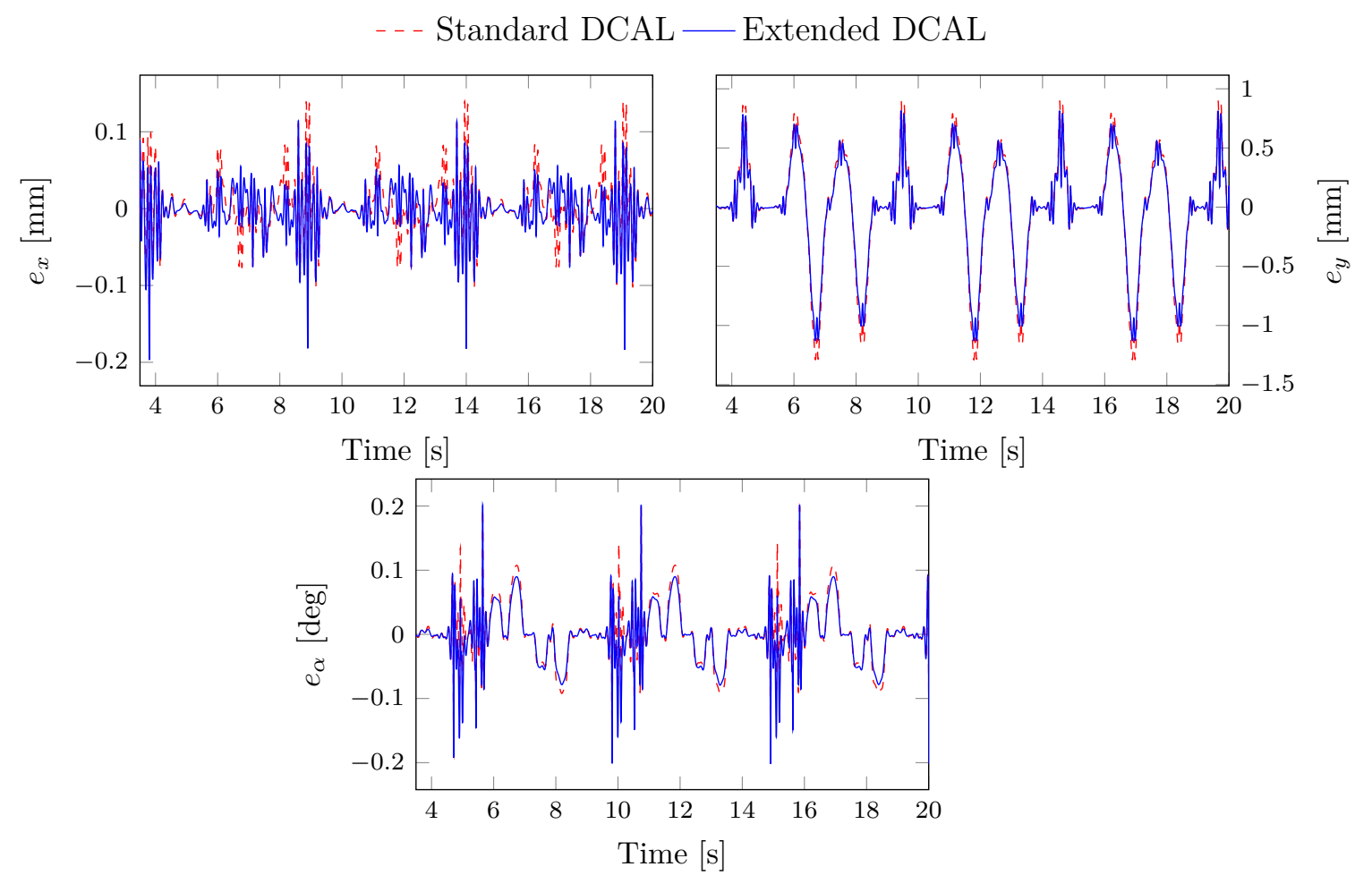

Figure 6. Scenario 1: evolution of the Cartesian tracking errors versus time

The estimated parameters' evolution is depicted in Figure 8. As it was expected, the estimated parameters converge in the case of standard DCAL since this controller is known for its good adaptation performance. The proposed modification does not hurt this feature as it can be observed from Figure 8. Indeed, the estimated parameters in both controllers converge within the same time span. Furthermore, it should be noticed that the steady state values of the estimated parameters (mass and the inertia of the moving platform) match those given by the CAD.

The control inputs are depicted in Figure 9. For the sake of clarity, the plot is zoomed to the interval $[11,14]$ seconds. We notice that the input torques generated by the proposed extended DCAL

Table 3. Scenario 1: evaluation and comparison of the tracking performance.

\begin{tabular}{lcc}
\hline & $\mathrm{RMSE}_{x y}[\mathrm{~m}]$ & $\mathrm{RMSE}_{\alpha}[\mathrm{deg}]$ \\
\hline Standard DCAL & $4.8173 \times 10^{-4}$ & $4.01 \times 10^{-2}$ \\
Extended DCAL & $4.1901 \times 10^{-4}$ & $3.63 \times 10^{-2}$ \\
Enhancement & $13 \%$ & $9.5 \%$ \\
\hline
\end{tabular}




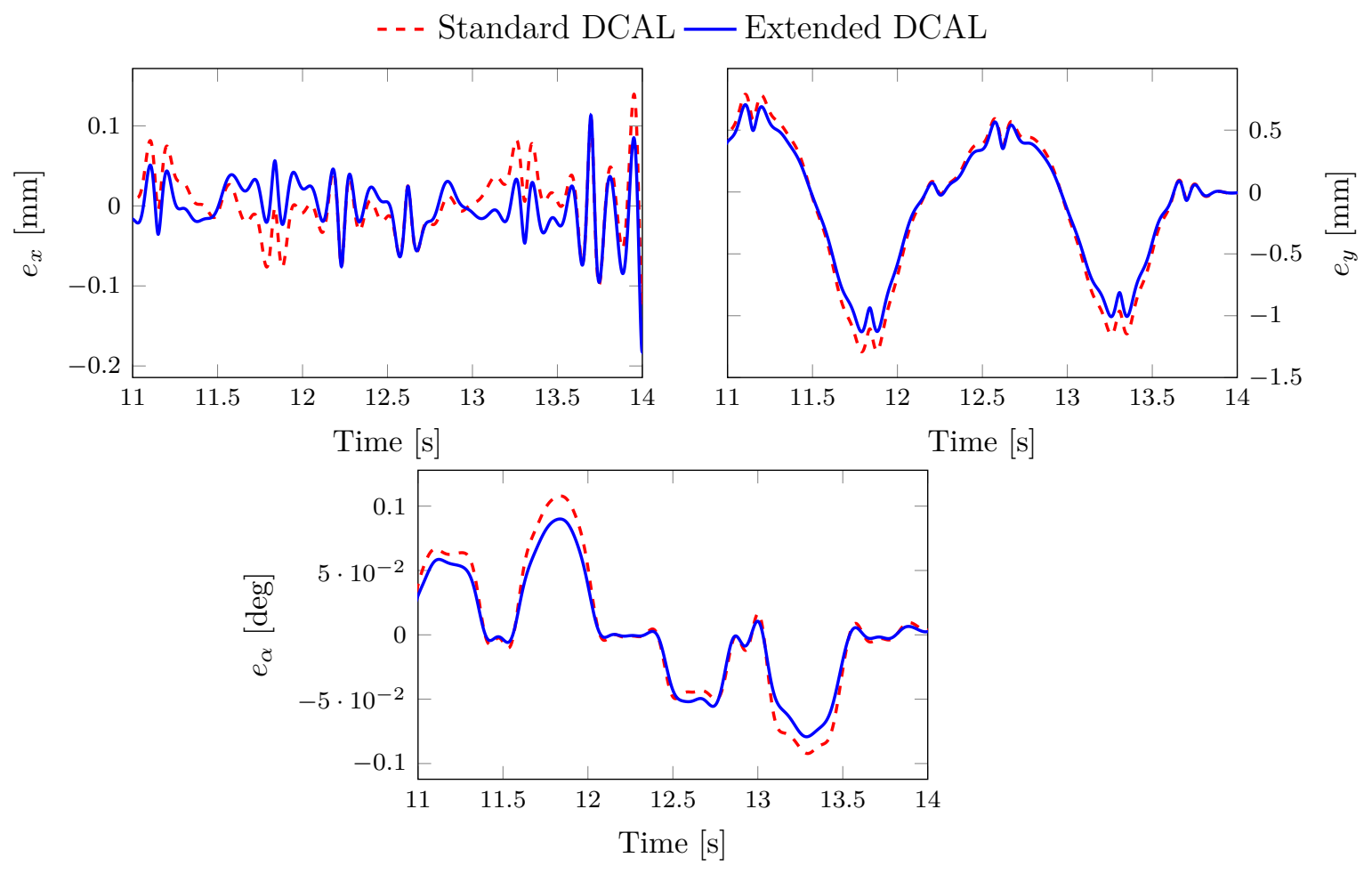

Figure 7. Scenario 1: zoom on the Cartesian tracking errors

controller are slightly reduced compared to standard DCAL. This is another feature of the nonlinear gains that worth to be highlighted.

\subsubsection{Scenario 2: payload handling}

In this scenario we added an extra payload to the moving platform of the robot with unknown mass and inertia. The motivation behind such scenario is to test the robustness of the controller toward uncertainties. In a real application, the manipulator is expected to handle different payloads with different masses. Hence, it is up to the control scheme to adjust its parameters to keep the tracking errors as small as possible.

Figure 10 depicts the Cartesian tracking errors of both controllers versus time. For clarity of the view, Figure 11 shows a zoome around the interval $[11,14]$ seconds of the Cartesian tracking errors for this scenario. Similar to the first scenario, the tracking errors are significantly reduced thanks to the use of nonlinear feedback gains in the control law. Table 4 summarizes the improvements brought by the proposed controller (up to 13.6\%) with respect to the original one.

The evolution of the estimated parameters for this scenario is shown in Figure 12. It can be observed that the adaptation algorithm adjusts the estimated parameters to newer steady state values. These new values correspond to the dynamic parameters of the moving platform including the additional payload.

The generated control inputs are depicted in Figure 13 from which it can be observed slight reduction of the energy consumption for the extended DCAL compared to the standard one. Furthermore,

Table 4. Scenario 2: Evaluation and comparison of the tracking performance.

\begin{tabular}{lcc}
\hline & $\mathrm{RMSE}_{x y}[\mathrm{~m}]$ & $\mathrm{RMSE}_{\theta}[\mathrm{deg}]$ \\
\hline Standard DCAL & $4.8726 \times 10^{-4}$ & $4.12 \times 10^{-2}$ \\
Extended DCAL & $4.2056 \times 10^{-4}$ & $3.74 \times 10^{-2}$ \\
Enhancement & $13.6 \%$ & $9.2 \%$ \\
\hline
\end{tabular}



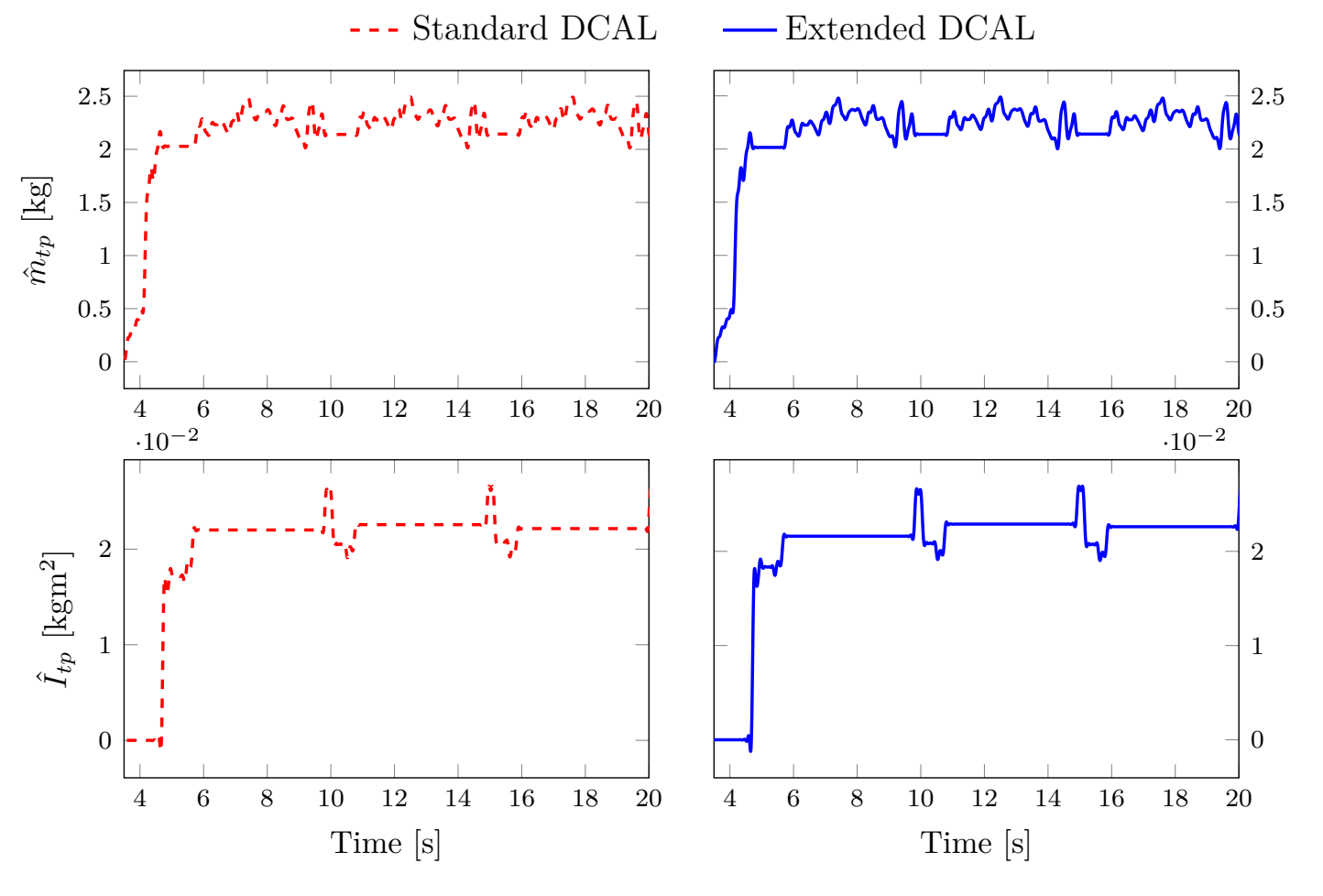

Figure 8. Scenario 1 - Evolution of the estimated parameters versus time

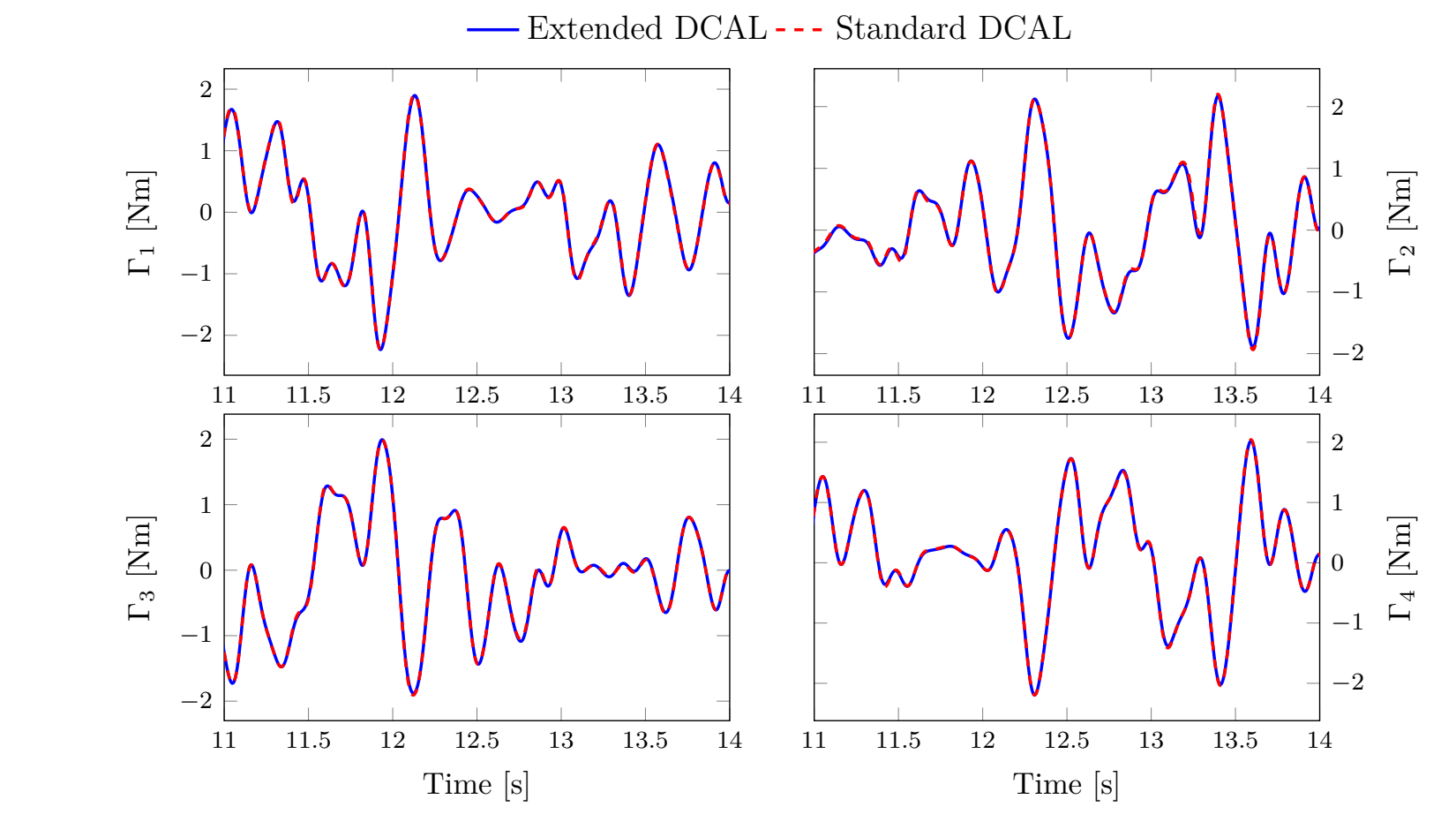

Figure 9. Scenario 1 - Evolution of the control inputs versus time

it can also be seen that, for both controllers, the generated torques remain within the admissible region since they do not exceed their maximum allowed values.

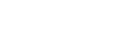

5

(1)

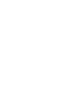




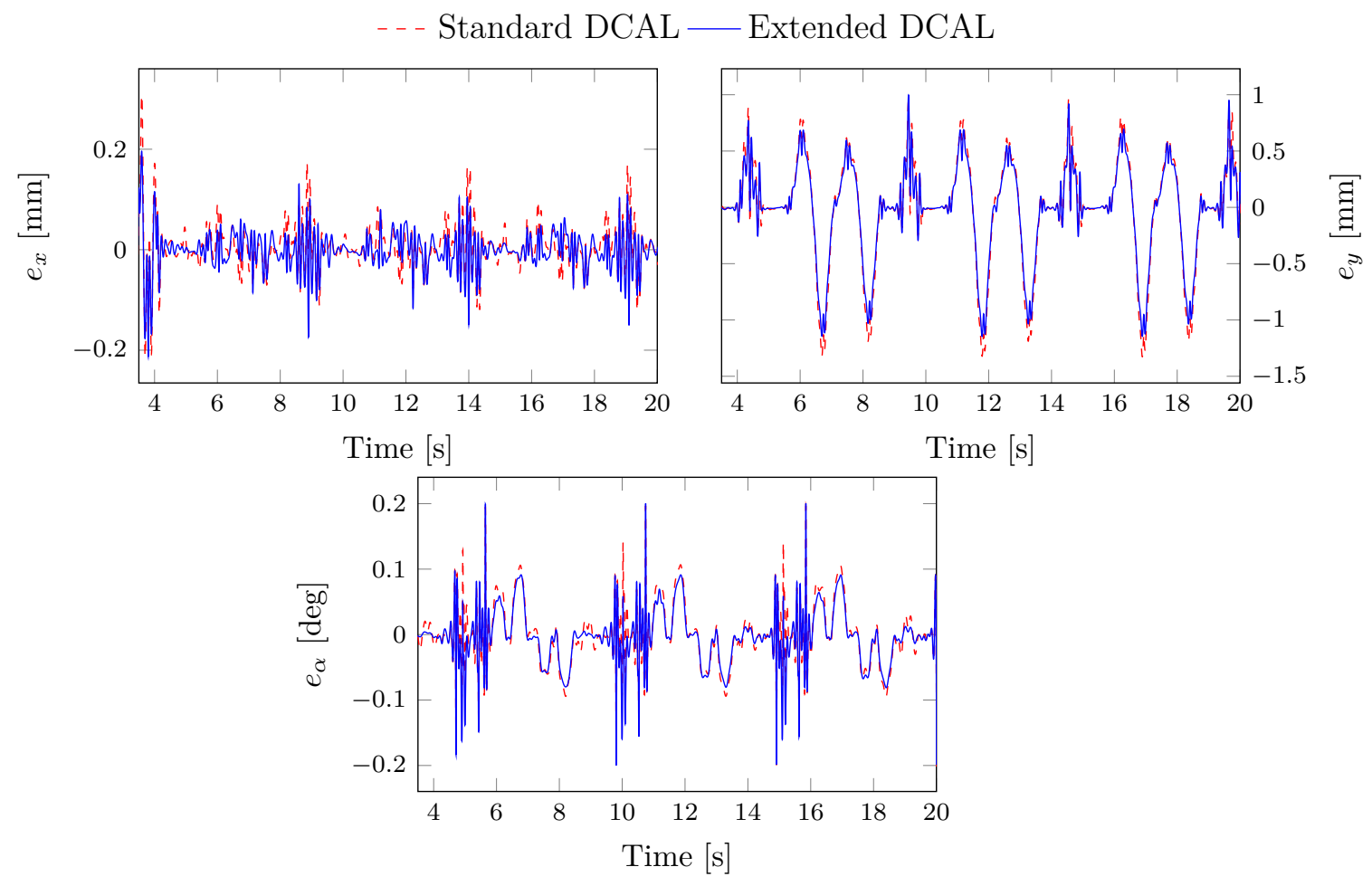

Figure 10. Scenario 2: evolution of the Cartesian tracking errors versus time

\section{Conclusions and Future Work}

In this paper, a new extension of DCAL controller was proposed. Several works in the literature have shown that using nonlinear feedback gains in different nonadaptive controllers has led to significant improvement of tracking performance. However, none of these works has demonstrated these advantages in the case of adaptive control, despite the fact that they often share structural similarities. Therefore, we have proposed in this work to revisit the DCAL adaptive controller by replacing its constant feedback gains by nonlinear time-varying ones. Since the choice of the nonlinear feedback gains is not unique, we have chosen a specific form for our gains that suits our needs. Specifically, the main feature of the proposed gains allow for fast tracking of large errors and a reduced overshot. Real-time experiments on our RA-PKM prototype have been used as a validation of the proposed control scheme. Indeed, the proposed extension significantly enhanced the closed-loop performance by reducing the tracking errors of the controller.

This work can be further extended by investigating other choices for the nonlinear gains and their optimization. Furthermore, we can consider analyzing the feasibility of including nonlinear gains in other adaptive control schemes to further demonstrate the relevance of such strategy.

\section{Acknowledgements}

This work was supported by the French National Research Agency, within the project ARROW (ANR-2011-BS3-006-01-ARROW).

\section{References}

[1] Merlet JP. Parallel robots, second edition. Dordrecht, Netherlands: Springer; 2006. 


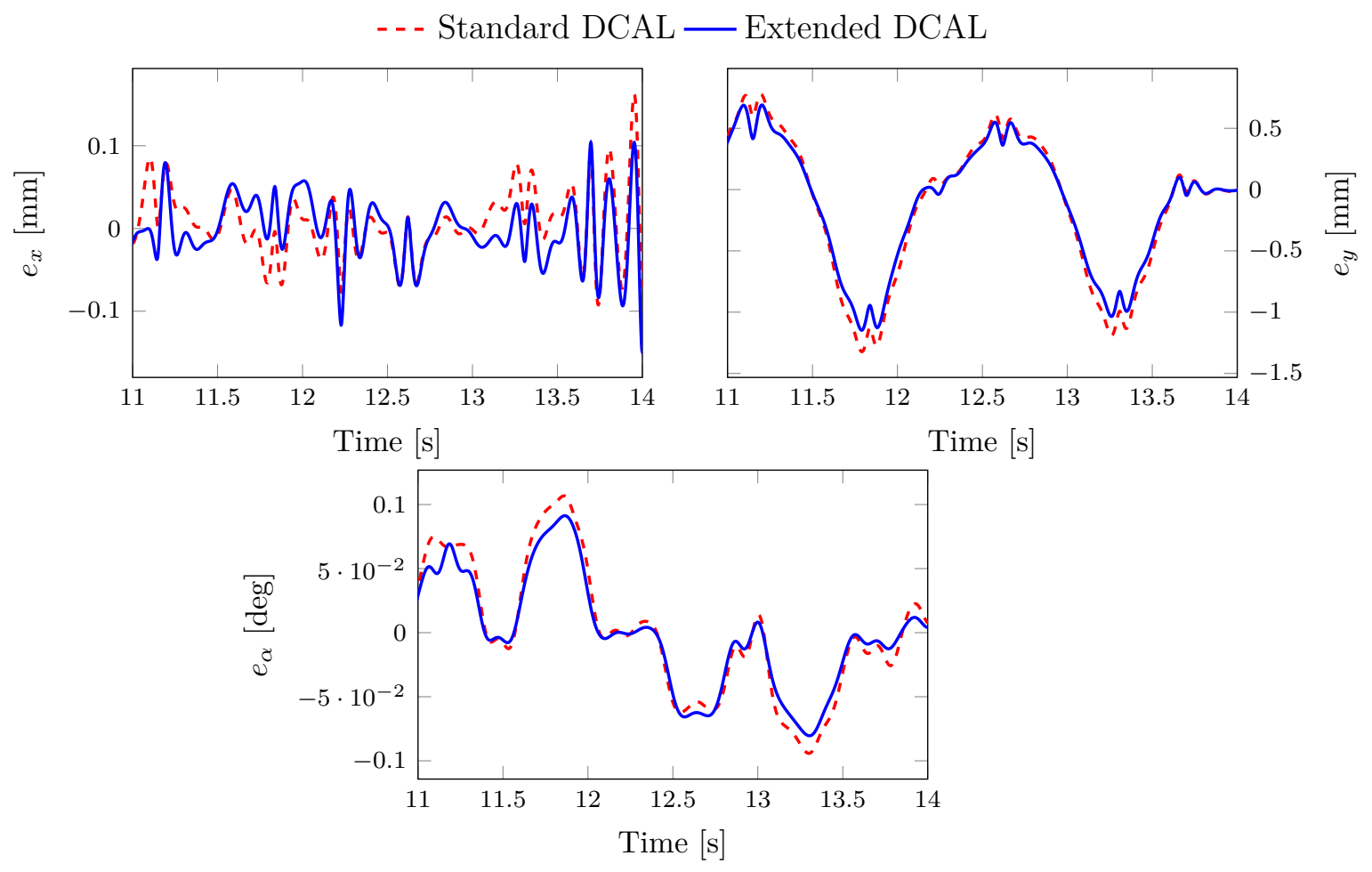

Figure 11. Scenario 2: zoom on the Cartesian tracking errors

- - - Standard DCAL — Extended DCAL
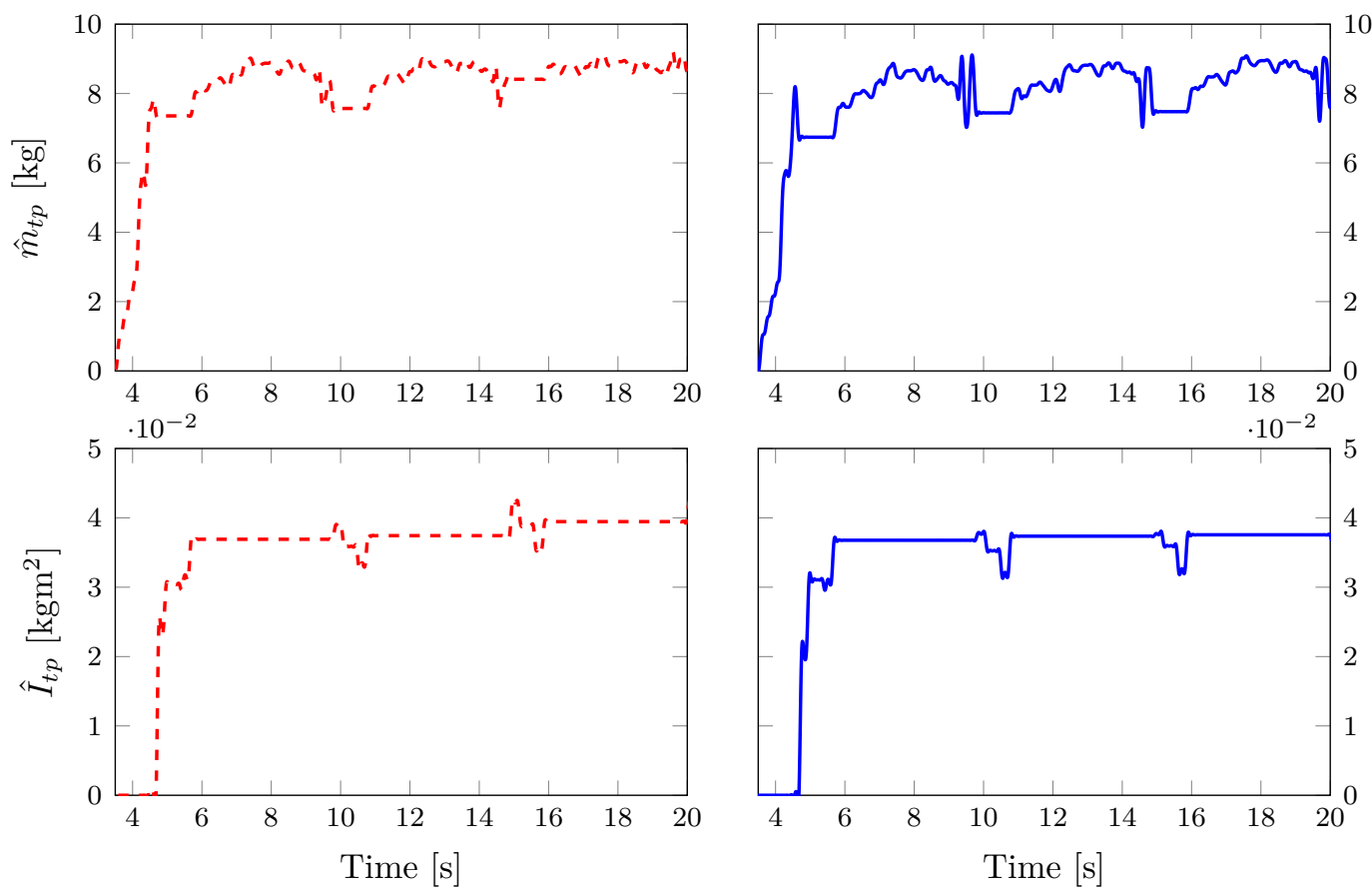

Figure 12. Scenario 2 - Evolution of the estimated parameters

[2] Lewis F, Dawson D, Abdallah C. Robot manipulator control: theory and practice. New York, USA: Marcel Dekker; 2003.

[3] Kiam HA, Chong G, Yun L. Pid control system analysis, design, and technology. IEEE Transactions on Control Systems Technology. 2005 Jul;13(4):559-576. 

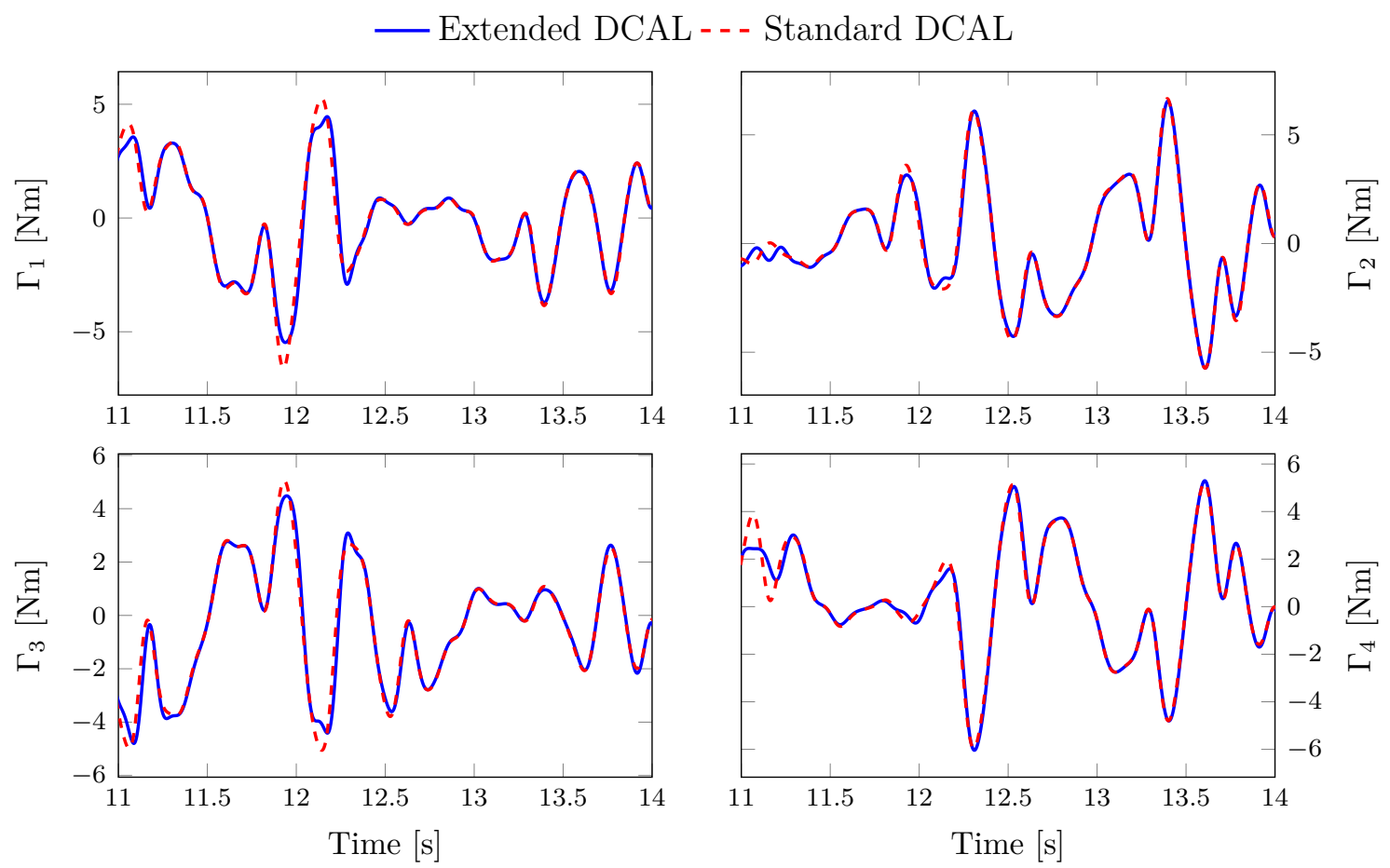

Figure 13. Scenario 2 - Evolution of the control inputs

[4] Han J. Nonlinear pid controller. Acta Automatica Sinica. 1994;04(20):487-490.

[5] Xu Y, Hollerbach J, Ma D. A nonlinear pd controller for force and contact transient control. IEEE Control Systems Magazine. 1995 Feb;15(1):15-21.

[6] Kelly R, Carelli R. A class of nonlinear pd-type controllers for robot manipulators. Journal of Robotic Systems. 1996 Dec;13(12):793-802.

[7] Seraji H. A new class of nonlinear pid controllers with robotic applications. Journal of Robotic Systems. 1998;15(3):161-181.

[8] Ouyang P, Zhang W, Wu F. Nonlinear pd control for trajectory tracking with consideration of the design for control methodology. In: IEEE International Conference on Robotics and Automation (ICRA'02); May; Vol. 4; 2002. p. 4126-4131.

[9] Cheng H, Yiu Y, Li Z. Dynamics and control of redundantly actuated parallel manipulators. IEEE/ASME Transactions on Mechatronics. 2003 Dec;8(4):483-491.

[10] Paccot F, Andreff N, P M. A review on the dynamic control of parallel kinematic machines: Theory and experiments. The International Journal of Robotics Research. 2009 Mar;:395-416.

[11] Luh J, Walker M, Paul R. Resolved-acceleration control of mechanical manipulators. IEEE Transactions on Automatic Control. 1980 Jun;25(3):468-474.

[12] Koditschek D. Natural motion for robot arms. In: IEEE Conference on Decision and Control (CDC'84); Dec.; 1984. p. 733-735.

[13] Paden B, Panja R. Globally asymptotically stable pd+ controller for robot manipulators. International Journal of Control. 1988;47(6):1697-1712.

[14] Craig JJ, Hsu P, Sastry SS. Adaptive control of mechanical manipulators. The International Journal of Robotics Research. 1987;6(2):16-28.

[15] Honegger M, Codourey A, Burdet E. Adaptive control of the hexaglide, a 6 dof parallel manipulator. In: Robotics and Automation, 1997. Proceedings., 1997 IEEE International Conference on; Apr.; Vol. 1; 1997. p. 543-548.

[16] Sadegh N, Horowitz R. Stability and robustness analysis of a class of adaptive controllers for robotic manipulators. The International Journal of Robotics Research. 1990 Jun;9(3):74-92.

[17] Shang W, Cong S. Nonlinear adaptive task space control for a 2-dof redundantly actuated parallel manipulator. Nonlinear Dynamics. 2010;59(1-2):61-72.

[18] Bennehar M, Chemori A, Pierrot F. A new extension of direct compensation adaptive control and its real-time application to redundantly actuated pkms. In: IEEE/RSJ International Conference on 
Intelligent Robots and Systems (IROS'14); Sep. Chicago, Illinois, USA; 2014. p. 1670-1675.

[19] Sartori Natal G, Chemori A, Pierrot F. Dual-space control of extremely fast parallel manipulators: Payload changes and the 100g experiment. IEEE Transactions on Control Systems Technology. 2015 Jul;23(4):1520-1535.

[20] Sartori Natal G, Chemori A, Pierrot F. Nonlinear control of parallel manipulators for very high accelerations without velocity measurement: stability analysis and experiments on par2 parallel manipulator. Robotica. 2014 Jun;:1-28.

[21] Shang W, Shuang C, Li Z, Long Jiang S. Augmented nonlinear pd controller for a redundantly actuated parallel manipulator. Advanced Robotics. 2009;23(12-13):1725-1742.

[22] Shang W, Cong S. Nonlinear computed torque control for a high-speed planar parallel manipulator. Mechatronics. 2009;19(6):987-992.

[23] Shang W, Shuang C. Nonlinear dynamic control and friction compensation of parallel manipulators. In: Kucuk DS, editor. Serial and parallel robot manipulators - kinematics, dynamics, control and optimization. InTech; 2009. p. 231-252.

[24] Khalil HK, Grizzle J. Nonlinear systems. Vol. 3. New Jersey, USA: Prentice hall; 1996.

[25] Slotine J, Li W. Applied nonlinear control. Prentice Hall; 1991.

[26] Muller A. Effects of geometric imperfections to the control of redundantly actuated parallel manipulators. In: IEEE International Conference on Robotics and Automation (ICRA'09); May; 2009. p. $1782-1787$.

[27] van der Wijk V, Krut S, Pierrot F, Herder JL. Design and experimental evaluation of a dynamically balanced redundant planar 4-rrr parallel manipulator. The International Journal of Robotics Research. 2013 Jun;32(6):744-759.

[28] Khalil W, Dombre E. Modeling, identification and control of robots. Kogan Page Science; 2004. 